\title{
Cough as the Presenting Symptom of Pancreatic Adenocarcinoma
}

\author{
R. Todd Richwine, DO, MS, Nadeem Abmed, MD, and Marta Mortensen, MD
}

Pancreatic cancer continues to be one of the most lethal of cancers. It is the 7 th leading cause of cancer in the United States and the 4th leading cause of cancer-related death. ${ }^{1}$ Although pulmonary metastases of pancreatic cancer is not uncommon, a nonproductive cough as the only complaint for greater than 6 months has not been previously reported.

\section{Case Reports}

A 49-year-old African American man presented to the family medicine clinic in July 2001 complaining of a persistent nonproductive cough not relieved by over-the-counter medications. He denied fever, chills, weight loss, pain, or other complaints. He continued to work at his job as a railroad engineer. The patient had a past medical history of hypertension, obesity, and hypercholesterolemia. He had never undergone surgery or been hospitalized. He had never smoked, nor had his wife, and he reported drinking less than 1 alcoholic beverage per month. There was no obvious history of exposure to infectious or occupational hazards. Family history was significant only for paternal cardiac disease. The patient's lungs were clear to auscultation, and results of the heart and abdominal examination were unremarkable. He did exhibit posterior pharyngeal nasal drip and clear rhinorrhea. Consequently, he was diagnosed with allergic rhinitis, treated with a nonsedating antihistamine, and told to return to clinic if he worsened.

In August 2001, his wife insisted that he return to the clinic. The patient's cough had not improved. A chart review indicated that the dosage of his angiotensin-converting enzyme (ACE) inhibi-

Submitted, revised 7 March 2003.

From the Forest Park Family Medicine Residency, Forest Park Hospital, St. Louis, Missouri. Address correspondence to R. Todd Richwine DO, MS, UNTHSC-Westside Family Practice, 5944 River Oaks Blvd., Fort Worth, TX 76114 (e-mail: rrichwin@hsc.unt.edu). tor was increased 1 month before his first visit for the cough. There were no changes in his physical examination, and his ACE inhibitor was discontinued. He continued without improvement and was evaluated by a second doctor in September 2001 . This physician documented that the patient's lungs continued to be clear to auscultation and found no other abnormalities on physical examination. The patient's chest radiograph (Figure 1) was read as having "diffuse nodular infiltrates seen throughout both lungs. Etiology unknown. However, due to the nodular nature, the possibility of metastatic disease cannot be ruled out entirely."

The chest radiograph was reviewed with the radiologist and a pulmonologist and a spiral computed tomograph (CT) was ordered (Figure 2). The spiral CT showed multiple bilateral nodules scattered throughout both lungs in all fields. No adenopathy was seen in either the hilar region or the rest of the mediastinum. No axillary or supraclavicular adenopathy was evident. Examination through the upper abdomen showed the visualized liver, spleen, suprarenal, and kidneys to be normal. The CT report stated "the differential diagnosis for scattered, diffuse bilateral pulmonary nodules includes sarcoidosis and fungal infections. [Tuberculosis] is a possibility but unlikely in proper settings. The appearance is not of metastasis or primary tumor."

Follow-up laboratory tests for ACE, HIV, and purified protein derivative were negative. Antinuclear antibody titers were negative, rapid plasma reagin was nonreactive, erythrocyte sedimentation rate was 60 , and cytidine monophosphate and complete blood cell count (CBC) were normal. The patient continued to have a bothersome cough, not even improved with narcotics at this point, and agreed to undergo a bronchoscopy in early October 2001. The bronchoscopy showed no abnormalities on visualization. Biopsies showed normal tissue bilaterally. Cultures for bacteria, fungus, and tuber- 

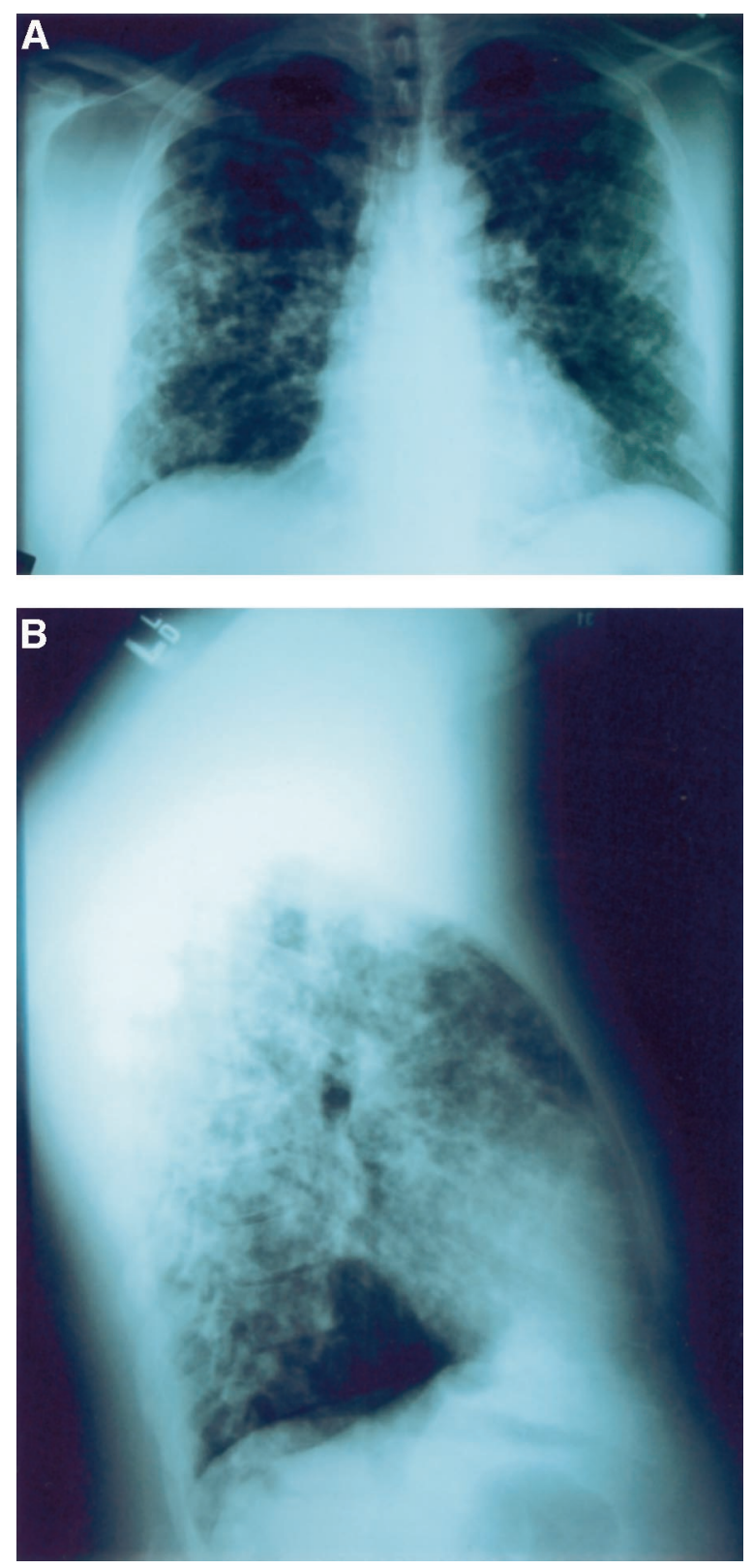

Figure 1. Anteroposterior (a) and lateral (b) radiographs show diffuse nodular infiltrates throughout both lungs.

culosis were negative. The patient declined videoassisted thoracostomy (VAT). Therefore, serial CTs and close follow-up was recommended.

Over the next 2 months, medical (bronchodilators, inhaled steroids, and high-dose codeine) and behavioral treatments failed to improve his cough, his examination remained unchanged, and he continued his job without difficulty. A repeat spiral CT of his chest showed "no significant change" in December 2001. The patient did not return for eval- uation until February 2002, when he called the clinic complaining of severe shortness of breath and dyspnea on exertion. He was immediately evaluated in the clinic, where he admitted to a significant worsening in his condition, and agreed to a VAT. His examination showed a 20-lb weight loss in the previous 2 months, and he appeared very fatigued. His cardiovascular examination was normal, his lung examination continued to be clear with no wheeze, rhonchi, or other abnormality. He had significant epigastric tenderness on palpation without rebound or guarding. Liver and spleen were of normal size.

He was admitted to our community hospital that day and labs were drawn. CBC and BMP continued to be normal except for an elevated $\mathrm{CO}_{2}$ of 30 $\mathrm{mEq} / \mathrm{L}$. Total bilirubin was elevated at $1.8 \mathrm{mg} / \mathrm{dL}$, alanine aminotransferase was 772 IU/L, aspartate aminotransferase was $347 \mathrm{IU} / \mathrm{L}$, amylase was 132 IU/L, lipase was $192 \mathrm{U} / \mathrm{L}$, alkaline phosphatase was $826 \mathrm{IU} / \mathrm{L}$, total protein was $7.8 \mathrm{~g} / \mathrm{dL}$, and albumin was $3.8 \mathrm{~g} / \mathrm{dL}$. Results of a hepatitis panel were negative, ESR continued to be $60 \mathrm{~mm} /$ hour, and rheumatoid factor was negative. Chest radiographs revealed increased densities of nodules in both lungs, with some associated inflammation or fluid. CT showed no significant change from the previous 2 CTs. Spiral CT with contrast of the abdomen and pelvis showed no abnormalities of the liver or spleen and no intrahepatic biliary ductal dilation. The pancreas and kidneys were read as normal. Because of the declining liver function, the VAT was canceled, and a gastroenterologist was consulted. A liver biopsy was performed and showed only mild acute hepatitis, with no evidence of tumor, metastases, or granulomatous inflammation. The patient continued to have a very slow increase in total bilirubin as the other liver enzymes slowly decreased, and he was released 3 days after admission pending the final liver biopsy results. The cultures of liver tissue were negative.

Two days after discharge, the patient, continuing to feel worse, was seen again. A repeat liver panel showed a doubling of the bilirubin and the patient was clearly jaundiced. His epigastric pain was now requiring high doses of oral narcotics. $\mathrm{He}$ was referred urgently to a GI specialist at a tertiary care center. He underwent an endoscopic retrograde cholangiopancreatography (ERCP), which showed a very tight stricture of the pancreatoduodenal duct and a small pancreatic head mass. A 


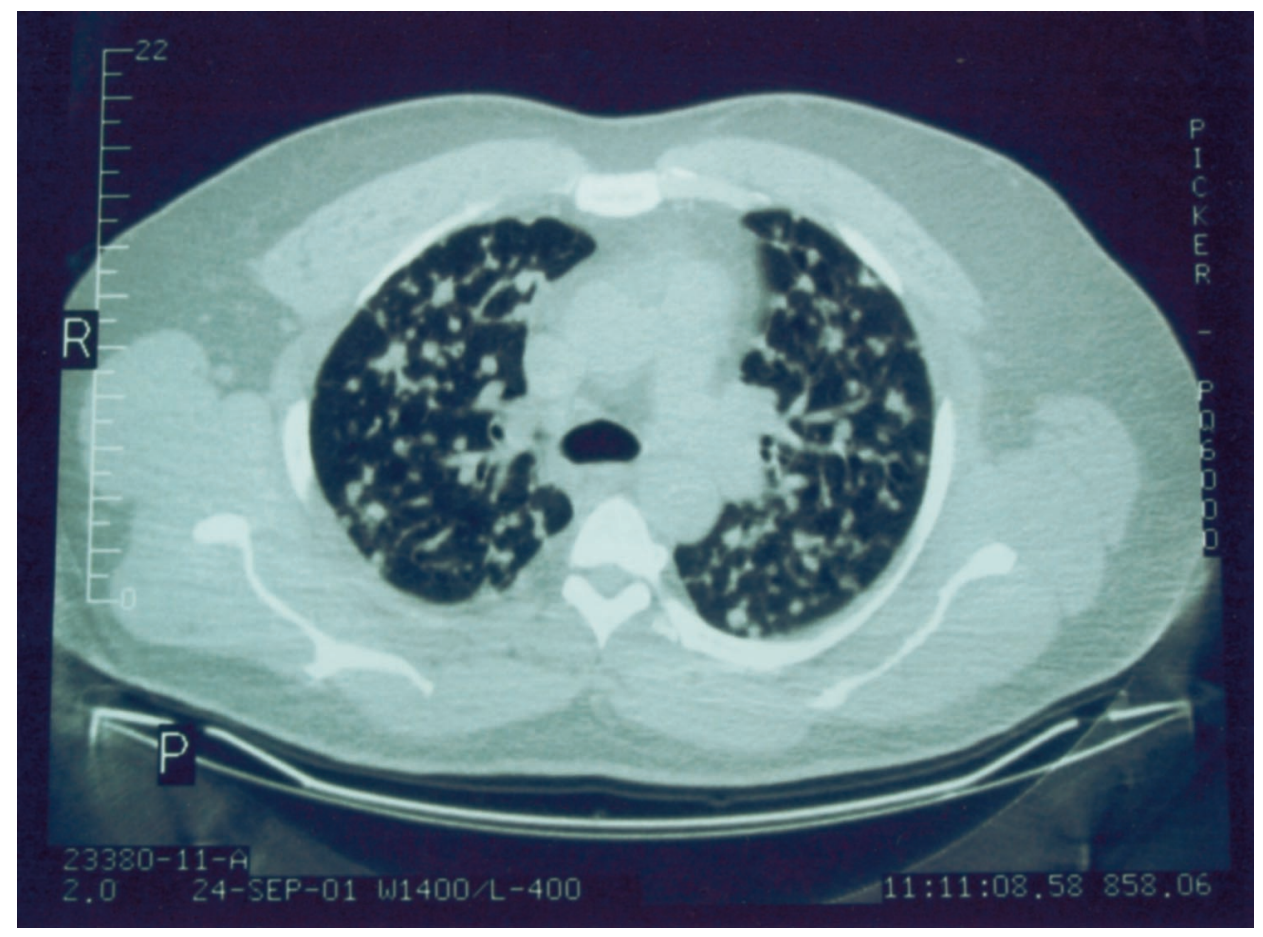

Figure 2. CT of lung fields shows multiple pulmonary nodules scattered throughout both lungs. These measured from a few millimeters to $1.5 \mathrm{~cm}$, No adenopathy is seen in either the hilar region or the rest of the mediastinum. No axillary or supraclavicular adenopathy is apparent.

stent was placed with considerable difficulty to relieve the obstruction. CA 19-9 level was then ordered and was found to be 39,900 (normal $<15$ ). A repeat spiral CT showed a small pancreatic duct mass with minimal but significant posterior peritoneal involvement. A positron emission tomography scan showed characteristic and identical signatures in the pancreatic head and the lung. He was diagnosed with metastatic, nonoperable pancreatic carcinoma. A CT-guided biopsy of a lung nodule further confirmed metastatic pancreatic adenocarcinoma.

\section{Discussion}

The incidence of pancreatic cancer rises above the age of $45 ; 80 \%$ of cases are found between the ages of 60 and 80 . Men are twice as likely as women to develop it, and mortality is increased in African Americans and Japanese Americans. Smoking is by far the most reducible risk factor accounting for up to $25 \%$ of pancreatic cancer in a dose-dependent relationship. Long-term alcohol consumption, high-fat diet, and chronic pancreatitis are more questionable risk factors. ${ }^{2}$ Pancreatic cancer continues to have the lowest 5 -year survival of any gastrointestinal malignancy. Only 1000 patients of the approximately 28,000 new diagnoses each year survive an additional 5 years. One-year survival from presentation is less than $20 \%$, and 6-month survival is less than $50 \%{ }^{3}$

Unfortunately, by the time patients present with symptoms they are often found to have local invasion or distant metastasis. The classic Virchow Triad of pancreatic cancer is typically found in masses in the head of the pancreas. The triad comprises abdominal pain, anorexia, and weight loss. Abdominal pain is typically epigastric, constant, dull, and may radiate to the back. Pain is often worse at night and when the patient is supine. Pain will often decrease when the patient leans forward, bringing the mass off the celiac plexus. ${ }^{4}$ Pain is found as the initial symptom in $70 \%$ to $80 \%$ of patients and is typically secondary to mass effect, obstruction of outflow from the liver, or local invasion of nerves. Anorexia and weight loss are the initial presenting symptoms in $10 \%$ of patients and are thought to be secondary to malabsorption and decreased caloric intake. Jaundice is a frequent and common symptom, usually secondary to obstruction of the biliary tree. Patients can develop diabe- 
Table 1. Findings in Pancreatic Adenocarcinoma at Initial Presentation

\begin{tabular}{lc}
\hline Symptoms/Findings & Frequency \\
\hline Abdominal pain & $70 \%-80 \%$ \\
Anorexia & $10 \%$ \\
Weight loss & $10 \%$ \\
Hepatomegaly or jaundice & $80 \%$ \\
Diabetes \& glucose intolerance & $10 \%-15 \%$ \\
Trousseau sign & $<10 \%$ \\
Courvoisier sign & $30 \%$ \\
\hline
\end{tabular}

tes, and glucose intolerance is thought to develop 6 to 12 months before diagnosis in $10 \%$ to $15 \%$ of patients. ${ }^{3}$ A physician should consider pancreatic cancer in patients with type I or atypical diabetes, who have no family history, are not obese, and who rapidly progress to insulin dependence. Superficial thrombophlebitis (Trousseau sign) was originally described in patients with pancreatic cancer but has been found in other cancers with equal prevalence. Courvoisier sign, a palpable gallbladder, is found in $30 \%$ of patients (Table 1$)^{2}$

When there is suspicion of pancreatic cancer, there are several diagnostic procedures that may be beneficial. The availability, cost, and ease of interpretation of helical (spiral) CT scanning favor it as the initial test of choice. Helical CT scans can detect focal masses, biliary dilation, hepatic metastases, ascites, nodal invasion, and vascular involvement. Helical CT scans can detect cancer and resectability with approximately $80 \%$ sensitivity but will miss tumors and metastases less than $2 \mathrm{~cm}$ in size. Ultrasound has a $70 \%$ sensitivity but is unable to stage the cancer or determine resectability. MRI is more expensive than CT without any increase in determining resectability. ERCP is another modality that offers increased sensitivity over CT (90\%), provides the ability to obtain biopsies, and can relieve obstruction by placing stents. ERCP, although beneficial, is both expensive and invasive. CA 19-9 is a tumor marker that should only be used in patients suspected of having pancreatic cancer. Levels correlate with tumor burden and level of cancer expression and are therefore often normal in small tumors. Approximately $15 \%$ of persons cannot synthesize this antigen and will never express high levels. CA 19-9 can be positive in jaundiced patients from biliary obstruction alone as in simple cholelithiasis. When the level is $>1000 \mathrm{U} / \mathrm{mL}$, specificity reaches nearly $100 \%$ but sensitivity drops to $40 \% .^{2}$

Pancreatic carcinoma frequently demonstrates metastatic lesions in the liver (62\%), the lung (55\%), and bone (25\%). ${ }^{5}$ These pulmonary metastases may mimic lung cancer, primarily bronchoalveolar carcinoma. ${ }^{6}$ Cassiere et $\mathrm{al}^{7}$ retrospectively studied 294 patients with pancreatic carcinoma and found lung cancer as the primary diagnosis in 10 of these patients (3.4\% of patients), all of whom were smokers. Tumors of the body and tail of the pancreas were more likely to mimic primary lung tumors than tumors of the head of the pancreas (about $10 \%$ vs $1 \%$ ). Nine of the 10 patients had solitary tumors; one had multiple diffuse lesions.

In a different study, Kamisawa et $\mathrm{al}^{5}$ investigated metastatic spreading from pancreatic carcinoma in 130 autopsy cases. Sixteen patients had pulmonary metastases without hepatic metastases as in the described patient, and 81 patients showed hepatic involvement. The average age of these patients was 65.6 years, and retroperitoneal invasion was found in 14 cases; lymph node metastases were present in all cases. Importantly, prolonged survival of patients in this group (mean $>10$ months) suggested that radiotherapy received by 11 of 16 patients affected some routes of metastatic spread.

Fewer than $20 \%$ of patients presenting with initial symptoms are candidates for surgery, the only curative treatment. A Whipple procedure combined with adjuvant chemotherapy can have cure rates near $25 \%$ but is unable to increase the number of cancers deemed resectable. Jaundice is often best treated by biliary stent placement, but stents often reocclude. As mentioned earlier, pain is primarily caused by invasion of the celiac plexus, with $90 \%$ of patients experiencing pain reduction. The most common side effect is postural hypotension. ${ }^{4}$ Primary physicians should always be vigilant for malabsorption (if it occurs, treat with pancreatic enzymes) and diabetes with rapid progression to insulin dependence (Figure 3$)^{2}$

\section{Conclusion}

We believe this to be the first reported case of pure pulmonary complaints as the presenting and continuing symptom of metastatic pancreatic cancer. Our patient did not present with any of the "classic Vichow Triad" until 8 months after initial presentation with a cough. His initial CT of chest and 


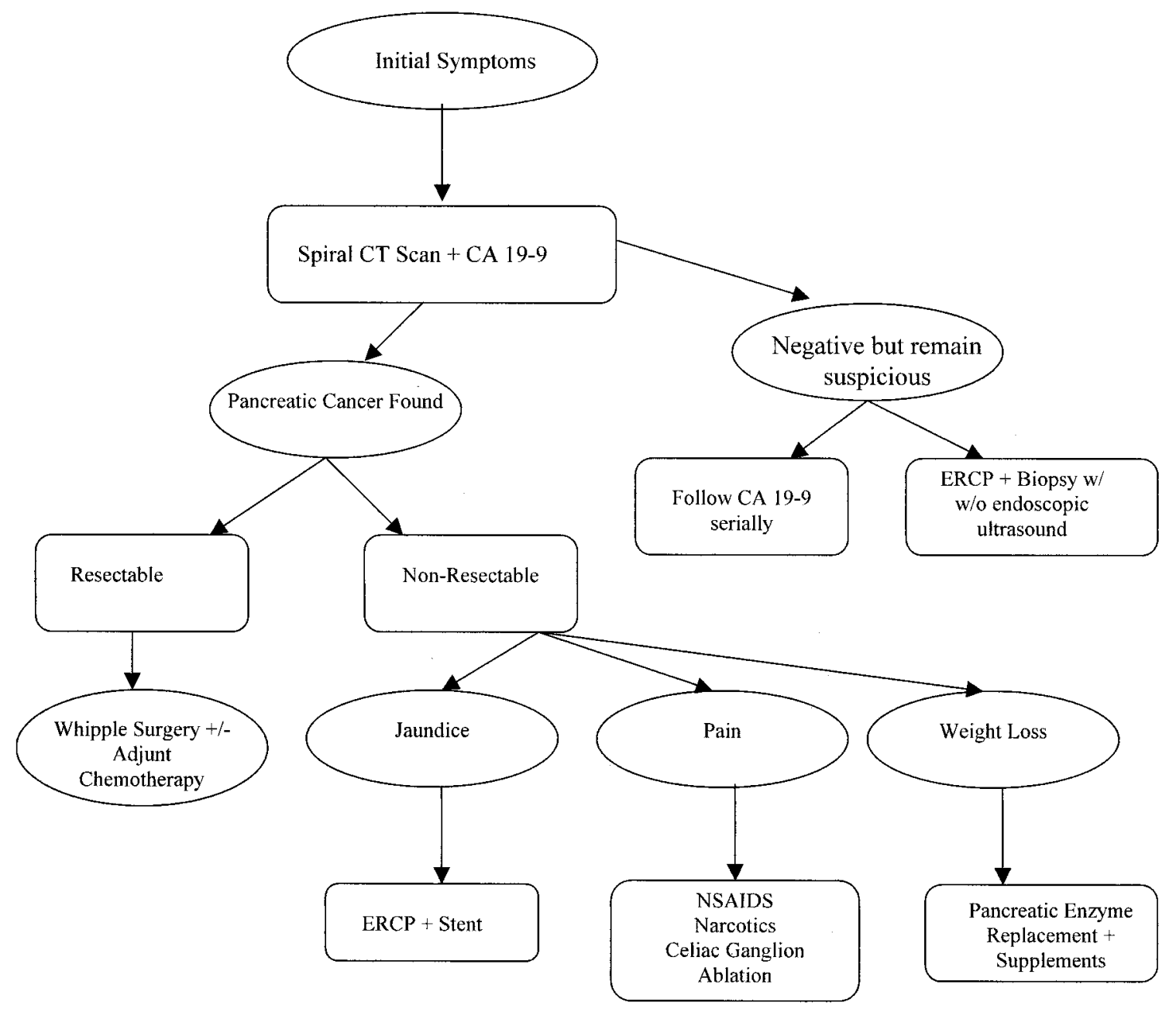

Figure 3. Diagnosis and management of pancreatic cancer.

bronchoscopy was nondiagnostic. Of the few cases found in the literature, ${ }^{5,6,9,10,11}$ our patient's lung metastases were multiple (not solitary), his cancer was in the pancreatic head (tail is $90 \%$ more common in patients with lung metastases), and he was never a smoker. Even after presentation with epigastric pain, a helical CT scan (the test of choice) was nondiagnostic. All the reported cases had other symptoms at presentation, metastases were found only at autopsy, or were previously diagnosed as having pancreatic cancer before they developed lung symptoms. It was not until the liver obstruction became severe enough to require an ERCP that carcinoma was considered. A CA 19-9 level was then ordered. A positron emission tomography scan, a test not readily available in nonspecialty centers, indicated the similar signal between the lung and pancreatic cancer.
Diagnosing pancreatic cancer is challenging for family practitioners. However, early diagnosis is the patient's only chance for a surgical cure. Our family practice clinic cares for 2 patients with incidentally detected cancers, one patient 5 years out from diagnosis and the other 10 years out. Physicians should always maintain a high index of suspicion for pancreatic cancer, especially in older patients. The classic triad of abdominal pain, anorexia with weight loss, and jaundice should never be ignored. Spiral CT scans with rapid contrast combined with a CA 19-9 level used in suspicious cases seems to be the most useful diagnostic tests and are also able to stage the cancer if found.

At the date of submission, the patient is receiving continual home oxygen and enjoying the time with his family. He is free from pain after a celiac ablation, is being treated by "salvage chemother- 
apy," and is currently 20 months from initial presentation.

\section{References}

1. Lehman GA, Uzer MF. Pancreatic cancer. Best practice of medicine.

2. Barkin JS, Goldstein JA. Diagnostic approach to pancreatic cancer. Gastroenterol Clin North Am 1999;28:709-22.

3. Cooperman AM. Pancreatic cancer: the bigger picture. Surg Clin North Am 2001;8:557-74.

4. Ahlgren H. Gastrointestinal malignancies. Prim Care 2001;28:647-60.

5. Kamisawa T, Isawa T, Koike M, Tsuruta K, Okamoto A. Hematogenous metastases of pancreatic ductal carcinoma. Pancreas 1995;11:345-9.

6. Steinke K, Suess K, Wiesner W. Pulmonary metastases from pancreatic adenocarcinoma mimicking bronchoalveolar carcinoma. Eur Radiol 2000;10: 1683-4.

7. Cassiere SG, McLain DA, Emory WB, Hatch HB. Metastatic carcinoma of the pancreas simulating primary bronchogenic carcinoma. Cancer 1980;46: 2319-21.

8. Leach WB. Carcinoma of the pancreas; a clinical and pathological analysis of thirty-nine autopsied cases. Am J Pathol 1950;26:333-47.

9. Bunker SR, Klein DL. Multiple cavitated pulmonary metastases in pancreatic adenocarcinoma. Br J Radiol 1982;55:455-6.

10. Gaeta M, Volta S, Scribano E, Loria G, Valllor A, Pandolfo I. Air-Space pattern in lung metastasis from adenocarcinoma of the GI tract. J Computer Assist Tomogr 1996;20:300-4.

11. Mishriki TT. Pancreatic carcinoma presenting as primary lung cancer. Postgrad Med 1990;87:107-10. 